\title{
The Mental Health of Pregnant Women During COVID-19
}

\author{
Poorya Davoodi ${ }^{1,2}{ }^{\oplus}$, Meisam Akhlaghdoust ${ }^{2,3^{*}}{ }^{\oplus}$ \\ ${ }^{1}$ Department of Molecular Medicine, University of Padua, Padua, Italy \\ ${ }^{2}$ Functional Neurosurgery Research Center, Shohada Tajrish Comprehensive Neurosurgical Center of \\ Excellence, Shahid Beheshti University of Medical Sciences, Tehran, Iran \\ ${ }^{3}$ USERN Office, Functional Neurosurgery Research Center, Shahid Beheshti University of Medical \\ Sciences, Tehran, Iran
}

*Correspondence to Meisam Akhlaghdoust, Email: akhlaghdoust@sbmu.ac.ir

Published online June 30, 2021

\section{Dear Editor,}

Severe acute respiratory syndrome coronavirus 2 (SARS$\mathrm{CoV}-2$ ) has become a global health emergency since the end of $2019 .{ }^{1}$ It has been the cause of more than 2.06 million deaths around the world until now. ${ }^{2,3}$ As you know, this pandemic has caused many problems and has had damaging effects on various aspects of people's lives. One of the essential issues to pay special attention to is women's mental health during pregnancy. In this letter, we would like to explain the immediate need to focus on the psychological impact of the SARS-Cov-2 during pregnancy.

Several reports have shown that the rate of psychopathology (anxiety, depression, etc.) in pregnancy has increased significantly during pandemics. This increasing rate was related to fear of infection with the virus, reduce support services in hospitals and operating rooms, inadequate prenatal care, and social isolation from support networks. ${ }^{4}$

Mortazavi and colleagues published an article regarding pregnant women's well-being and worry during the COVID-19 pandemic. ${ }^{5}$ The results of their study showed that the COVID-19 pandemic has had a harmful effect on pregnant women in terms of mental health. A little percentage of pregnant women have severe coronavirus infection, but this pandemic has psychological consequences for the neonate and the mother.

Perinatal support is a service for any woman with mental health problems, who are planning a pregnancy, are pregnant, or have a baby up to one year of age. There has been a significant decrease in prenatal care around the world, which could lead to increased rates of psychopathology problems which is in turn a risk factor for poor postpartum health and postnatal depression. Since the start of the pandemic, for the protection of pregnant women and their children, only one person is allowed to enter the delivery room and unnecessary visits are unacceptable.

Perinatal support leads to reduced pain during delivery, increases psychological health, and satisfaction during pregnancy, so it seems that these policies have some harmful effects, and they will show themselves in the near future.

Moreover, during the pandemic, violence against women has increased as a result of economic stress and financial instability. Domestic violence is closely related to psychopathology, especially increased rates of depression, anxiety, and suicide. During pregnancy, mental health disorders can have very serious consequences for both the mother and baby. For instance, increased perinatal psychopathology can result in the usage of drugs, alcohol, and future suicide. In addition, when the mother has stress, many problems can restrict fetal growth, lead to premature birth, and induce low birthweight.

According to all the explanations above, future research should aim to recognize women who are exposed to domestic violence, as well as the psychological effects of the pandemic on pregnant women and their children. To solve these problems, careful planning and management is needed and if not addressed, it can lead to irreversible outcomes in the future.

In order to have a healthy society in future, we also suggest that after the end of this pandemic we need to set up a screening and control program for women who have given birth during this pandemic to monitor and manage possible consequences.

\section{Conflict of Interest Disclosures}

The authors declare that they have no conflict of interests.

Ethical Statement

Not applicable. 


\section{References}

1. Moazzami B, Chaichian S, Samie S, Majidi Zolbin M, Jesmi F, Akhlaghdoust $M$, et al. Does endometriosis increase susceptibility to COVID-19 infections? a case-control study in women of reproductive age. BMC Womens Health. 2021;21(1):119. doi: 10.1186/s12905-021-01270-z.

2. Abba-Aji A, Li D, Hrabok M, Shalaby R, Gusnowski A, Vuong W, et al. COVID-19 pandemic and mental health: prevalence and correlates of new-onset obsessivecompulsive symptoms in a Canadian province. Int J Environ Res Public Health. 2020;17(19):6986. doi: 10.3390/ ijerph17196986.

3. WHO. COVID-19 weekly surveillance report.
Https://WwwEuroWhoInt/En/Health-Topics/ Health-Emergencies/Coronavirus-Covid-19/WeeklySurveillance-Report. 2020;2021(December 2020):2021.

4. Corbett GA, Milne SJ, Hehir MP, Lindow SW, O'Connell $\mathrm{M}$ P. Health anxiety and behavioural changes of pregnant women during the COVID-19 pandemic. Eur J Obstet Gynecol Reprod Biol. 2020;249:96-7. doi: 10.1016/j. ejogrb.2020.04.022.

5. Mortazavi F, Mehrabadi M, Kiaeetabar R. Pregnant women's well-being and worry during the COVID-19 pandemic: a cross-sectional study. BMC Pregnancy Childbirth. 2021;21(1):59. doi: 10.1186/s12884-021-03548-4.

Citation: Davoodi P, Akhlaghdoust M. The mental health of pregnant women during COVID-19. Clin Neurosci J. 2021;8(3):103-104. doi:10.34172/icnj.2021.22. 